\title{
A fascinating overview of the biology of fragile $X$ syndrome
}

\author{
Martin Hergersberg ${ }^{1}{ }^{1}$ \\ Received: 12 July 2018 / Accepted: 19 July 2018 / Published online: 3 September 2018 \\ (c) European Society of Human Genetics 2018
}

Fragile X syndrome: from genetics to targeted treatment Editors: Rob Willemsen, R. Frank Kooy

Published by: Academic Press (Elsevier)

Year: 2017

ISBN: 9780128044612 (Hardcover), 9780128045077

(eBook)

Price: $£ 82.60 \quad € 102.40 \quad \$ 105.00 \quad$ (Hardcover), $£ 99.12$

$€ 116.89 \$ 105.00$ (eBook)

This fascinating book, edited by Rob Willemsen and Frank Kooy, summarizes the current knowledge of the molecular aspects of fragile X syndrome (FXS), and the potential for therapies resulting from this knowledge. The appearance of the book in the $25^{\text {th }}$ anniversary year of the identification of the FMRI gene is a nice coincidence, as Ben Oostra remarks in his introduction.

The book is divided in three parts. The first part comprises seven chapters on the clinics, genetics, diagnosis, epidemiology, molecular mechanisms, pluripotent stem cell models and animal models of FXS. Because the focus of the book is on potential target-specific pharmaceutical treatments for different symptoms of FXS, the second part, "Pathways involved," is the largest part of the book. It comprises ten chapters: on target molecules of the FMR protein, on the mGluR theory of FXS, on the GABAergic system, intracellular signaling networks, the endocannabinoid system, glycogen synthase kinase-3, Rho GTPase signaling, matrix metalloproteinases, ion channels, and reactivation of the FMR1 gene. The third part, "Clinical trials," contains chapters on targeted pharmacotherapy, and on different aspects of targeted clinical trials in FXS. Particularly interesting is a review "fragile X research from a parental perspective," which summarizes the results of an international survey of the attitudes of families with FXS relatives towards research.

Martin Hergersberg

Martin.Hergersberg@zlmsg.ch

1 Centre for Laboratory Medicine, Frohbergstr. 3, 9001 St Gallen, Switzerland
Although many clinical trials for a therapy for FXS were performed, no treatment was identified yet. This is disappointing for the families participating in the trials, in particular as FXS was touted "poised to be the first neurobehavioral disorder in which corrective treatments have been developed from the bottom up" in 2011. Also discussed are the pronounced placebo effects observed during several clinical trials, which made it difficult for the families involved to accept that the trials are not resulting in a treatment.

This book is also an essential reference book for genetic counselors. They usually have a close personal contact to families with FXS patients. Often it is their task to accompany the families during clinical trials. The book provides counselors with the necessary biological background for proper explanations to the relatives of FXS patients. Many parents acquire a substantial knowledge of the conditions afflicting their children. The book will be helpful for both families and counselors to manage the expectations of families participating in clinical trials.

The only minor mistake I have noticed is the lack of a separate heading for "Drosophila" in the chapter on animal models. The information on this important animal model is therefore found in the section "Zebrafish." As in every multi-author book, there are some redundancies, but they are welcome, because informations are densely packed throughout the book.

In conclusion, this book is essential for everyone who is involved in the diagnosis and treatment of FXS patients.

\section{Compliance with ethical standards}

Conflict of interest The authors declare that they have no conflict of interest. 